\title{
Im Schwebezustand: Schulen und transgressive Lebenswelten Ansätze in Forschung und Lehre
}

\section{Annedore Prengel}

In der erziehungswissenschaftlichen Debatte um Interkulturalität und ihre Bedeutung für Schule und Lehrerausbildung gewinnt die Aufmerksamkeit für kontinuierlich dynamische Veränderungsprozesse an Bedeutung. Migration wird weniger als ein abgrenzbarer Schritt des Wanderns von einem Herkunftsland in eine neue Heimat, sondern als anhaltender Schwebezustand gedacht: Menschen wandern ein und wandern weiter, Kinder und Jugendliche bezeichnen ihr Leben als "dazwischen sein».

Der Beitrag stellt bildungstheoretische, empirische und pädagogisch-praktische Ansätze der erziehungswissenschaftlichen Auseinandersetzung mit Transnationalität vor. Beziehungen zu anderen disziplinären Feldern (z.B. "Transgender" und "Transitionen" im Bildungssystem) werden hergestellt. Abschliessend werden Schlüsse für die Weiterentwicklung der "Pädagogik der Vielfalt" und ihre Konkretisierung in Konzepten der Forschung und der Ausbildung für Lehrpersonen von der Elementarstufe an gezogen.

Schulen werden von Kindern besucht, deren Leben von den Veränderungsprozessen in ihren Lebenswelten beeinflusst ist. In der erziehungswissenschaftlichen Debatte um Heterogenität und ihre Bedeutung für Schule und Lehrerausbildung gewinnt die Aufmerksamkeit für kontinuierlich dynamische Veränderungen im Lebenszusammenhang der Kinder ${ }^{1}$ an Bedeutung. Migration wird nicht nur als ein abgrenzbarer Schritt des Wanderns von einem Herkunftsland in eine neue Heimat, sondern als ein anhaltender Schwebezustand wahrgenommen: Menschen wandern ein und wandern weiter, Kinder und Jugendliche bezeichnen ihr Leben als «dazwischen sein».

Der folgende Beitrag bezieht sich auf einen aktuellen Ausschnitt der erziehungswissenschaftlichen Zeichnung eines Bildes vom Kind, in dem das Phänomen der «Transnationalität» und anderer Transgressionen ${ }^{2}$ berücksichtigt wird. Er untersucht die Frage, welches Bild vom Kind aus der mit der Betonung des Veränderlichen verbundenen Einsicht in den transgressiven Charakter aktueller Lebenswelten hervorgeht. Die Studie stellt bildungstheoretisch, empirisch und 
pädagogisch-praktisch orientierte Ansätze der erziehungswissenschaftlichen Auseinandersetzung 3 (Wigger, 2003; Koller, 2002) mit Transgressionalität vor. Nach einer kurzen einleitenden Klärung erkenntnistheoretischer Voraussetzungen werden in einem ersten Schritt Analysekategorien und ihre Überschneidungen zur Diskussion gestellt; im zweiten Schritt wird die Bedeutung von Transgressionen in mehreren disziplinären Feldern analysiert; im dritten Schritt werden Schlüsse für die Weiterentwicklung der «Pädagogik der Vielfalt» und für die Ausbildung von Lehrpersonen von der Elementarstufe an gezogen.

Der erziehungswissenschaftliche Blick auf Kinder in Schulen hat immer neue Bilder von Kindern hervorgebracht. Seit den Kinderbeobachtungen aus den Anfängen der Erziehungswissenschaft im 18. Jahrhundert (Tenorth, 2004) unterscheiden sich diese Vorstellungen über Kinder je nach historischen Kontexten und theoretischen Strömungen. Die aktuelle pädagogische Anthropologie weist darauf hin, dass ein Anspruch Aussagen über "den Menschen» und damit auch über "das Kind» zu machen, prinzipiell nicht einlösbar wäre. «Derartige universalistische Ansprüche bedürfen historischer, kultureller und epistemologischer Relativierung» (Wulf 2004, S. 40). Darum sind vielfältige wissenschaftliche Aussagen und Aussagesysteme über Kinder in Schulen unumgänglich. Zweifach sind sie historisch und kontextuell bedingt, weil zwei Personengruppen unmittelbar beteiligt sind: Schülerinnen und Schüler auf der einen und Forscherinnen und Forscher auf der anderen Seite. Aus dem Treffen beider Seiten im Forschungsprozess gehen die Erkenntnisse hervor. Wenn Studien ihr Augenmerk auf Transgressionen, auf Übergänge im Bildungsprozess richten, so beziehen sie sich vor allem auf Bewegungen der Kinder und ihres gesellschaftlichen Umfeldes, auf Veränderungen im Laufe der Zeit. Die Übergänge von Kindern, mit denen Schule zu tun hat, können durch sehr unterschiedliche Bedingungen ihres Lebens begründet sein und müssen differenzierend untersucht werden. Solche Lebensbedingungen sind Zugehörigkeiten zu unterschiedlichen gesellschaftlichen Gruppierungen die u.a. durch Nationalität, Kultur, Schicht, Geschlecht, Alter und Fähigkeit bestimmt sind. Aus der Annahme, dass hier von relevanten Lebensbedingungen die Rede ist, werden Analysekategorien gebildet. Mit der Metapher des Reliefs zeigt Graumann (1960, 2002), wie die Prägung des forschenden Blicks jeweils einige Aspekte des Weltausschnitts in den Vordergrund, andere in den Hintergrund treten lässt. Er regt so dazu an, sich bewusst zu machen, dass die Art der Fragen und Kategorien mit denen sich die Forschenden dem ausgewählten, zu erkennenden Weltausschnitt annähern - hier Kinder in der Schule - die Art der Erkenntnisse erst ermöglicht. Für Untersuchung von Transgressionen ist charakteristisch, dass sie zwei Dynamiken verbindet: Forschende bewegen sich mit mehreren Analysekategorien suchend durch den gewählten Weltausschnitt «Kinder in der Schule» und dieser Weltausschnitt selbst ist von Veränderlichkeit bestimmt. 


\section{Changieren der Kategorien und Intersektionalität}

Disziplinäre Felder wie die auf Nationen, Kulturen und Ethnien, Geschlechterverhältnisse bezogenen Gebiete der Erziehungswissenschaft arbeiten mit Analysekategorien, die dazu dienen, relevante Befunde zum Leben und zum Lernen der Kinder in der Schule zu finden und zur Diskussion zu stellen. Sie sind zugleich verbunden mit gesellschaftlichen Machtkämpfen. Die Kategorienbildung ist heftig umstritten.

International läst sich beobachten, wie in sozialen und pädagogischen Bewegungen verwendete Begriffe changieren, sie betonen einmal die Gleichheit, ein andermal die Besonderheit ihrer Bezugsgruppe. Zu umgewerteten Begriffen in Kämpfen um Anerkennung gehören unter anderen «Krüppel», «Weiber», «Kanaken», «Queer», «Schwule» und «Lesben». Wechselnde Bezeichnungen für pädagogische Ansätze spiegeln diese Dynamik wider, zum Beispiel wenn von der «Ausländerpädagogik» über «Interkulturelle Pädagogik» zur «Migrationspädagogik» und «transkulturellen Pädagogik» gewechselt wird. Martin Fuchs (1999) hat den Wechsel zwischen der Betonung der Gleichheit und der Betonung der Differenz am Beispiel der indischen äusserst benachteiligten Kastenlose der «Dalit» untersucht. In Kämpfen um gleiche Rechte kann eine Tendenz zum Assimilationszwang, in Kämpfen um Anerkennung des Besonderen eine Tendenz zur idealisierenden Identifikation mitschwingen. Ich habe angesichts dieser Diskussion die Bedeutung der Menschenrechte für die Interpretation von Heterogenität in der Erziehungswissenschaft herausgearbeitet (Prengel, 2007a): Die Menschenrechte betonen in ihrer allgemeinen und in ihren gruppenbezogenen Fassungen die universelle Gleichheit aller Menschen, die nur möglich ist durch die universelle Freiheit aller Menschen (Bielefeldt, 1998). Heterogenität ist unmittelbar auf Egalität und Liberalität bezogen, denn es geht um die Freiheit für Verschiedenheit der Lebensentwürfe - eine Freiheit, die jedem Menschen zusteht und die Freiheit für neue Entwicklungen in der Zukunft einschliesst. Die Theorie der Menschenrechte und der Menschenrechtsbildung mit ihrer Betonung der gleichen Freiheit vermag es, einen gemeinsamen theoretischen Horizont für die Auseinandersetzung mit einzelnen Heterogenitätsdimensionen, auch in der Pädagogik, bereitzustellen.

Sozialwissenschaftliche (einschliesslich der erziehungswissenschaftlichen) Kategorienbildungen sind in die Kämpfe um Anerkennung verwickelt, sie werden von ihnen beeinflusst und beeinflussen sie ihrerseits. Stark vereinfachend und Ungleichzeitigkeiten vernachlässigend lässt sich eine Entwicklung der Erkenntnisinteressen und -stände hinsichtlich der meist berücksichtigten Heterogenitätsdimensionen beobachten: Sie reicht von einer Betonung der assimilatorisch interpretierten Gleichheitsrechte über die Betonung der aufwertend interpretierten Differenzerfahrungen und ihre Dekonstruktion zur Aufmerksamkeit für sich überschneidende Differenzdimensionen und für Überschreitungen von Differenzkonstruktionen. Während einige Teilnehmer der Debatten jeden neuen Er- 
kenntnisschritt über die Abwertung des vorangehenden legitimieren (vgl. z.B. in Teilen den Text von Mecheril, 2004), möchte ich vorschlagen, zu realisieren, dass jede neue Perspektive erst möglich wird auf der Basis des Erkenntnisstandes einer vorangehenden Perspektive sowie der Analyse ihres Gewinns und ihrer (unumgänglichen) Begrenztheit. So lässt sich für die stark international beeinflussten Debatten seit der westdeutschen Bildungsreform schlagwortartig eine Kettenreaktion beschreiben: Chancengleichheit - egalitäre Differenzen - ihre Dekonstruktion - ihre Zusammenführung im Intersektionalitätsdiskurs - Aufmerksamkeit für Transitionen, alle bauen aufeinander auf. Mataphern wie zum Beispiel «Differenzlinien» (Lutz \& Wenning, 2001) oder «Achsen der Ungleichheit» (Klinger, Knapp \& Sauer, 2007) dienen dazu komplexe Verhältnisse zu veranschaulichen.

Der Intersektionalitätsansatz regt dazu an, forschend nach der Relevanz von Geschlechts-, Kultur-, Schicht- und anderen Zugehörigkeiten zu fragen und zu berücksichtigen, dass Kinder zahlreichen Gruppierungen gleichzeitig angehören. Verzichtet man auf solche Dezentrierungen und bleibt bei der Arbeit mit einer Differenzkategorie, so ist das nur um den Preis starker Vereinfachungen möglich. Eine Annäherung an Kinder in der Schule im Sinne von Intersektionalität ermöglicht sehr differenzierte Erkenntnisse. In einer bildungstheoretisch fundierten Empirie (Wigger, 2003; Koller, 2002) kann man dafür offen sein herauszufinden, dass nicht einfach Jungen schlechte Schüler sind (das wäre eine unzulässige Universalisierung), sondern dass ökonomisch arme Jungen mit Migrationshintergrund und unzureichenden Bindungs- und Bildungserfahrungen häufig zur Gruppe der Schüler mit Schuldistanz gehören, dass aber auch Mädchen mit den gleichen Lebensbedingen Schuldistanz entwickeln können und dass Jungen und (in geringerer Anzahl) Mädchen einheimischer Herkunft, wenn sie soziokulturell benachteiligt sind und keine positiven Bindungs- und Bildungserfahrungen machen konnten, von Schuldistanz betroffen sind (Becker, 2007).

Leslie Mc Call (2005) unterscheidet drei Zugänge der Intersektionalität: Im antikategorialen Ansatz wird die Gefahr der Essentialisierung von Kategorienbildungen überhaupt hervorgehoben, im intrakategorialen Ansatz wird die Notwendigkeit innerhalb einer Kategorie mit Hilfe weiterer Kategorien zu differenzieren hervorgehoben, im interkategorialen Ansatz werden die Beziehungen zwischen verschiedenen Kategorien untersucht. Der letztgenannte ist nach Knapp (2005) der schwierigste und interessanteste Ansatz. Für die Schulpädagogik sind hier Fragestellungen angesprochen, die danach suchen, wie es dazu kommt, dass Geschlecht und Schulleistung teilweise so aufeinander bezogen sind, dass die Gruppe der schulversagenden Jungen und die Gruppe der schulerfolgreichen Mädchen grösser sind, obwohl immer auch Angehörige beider Geschlechter zu den mit und ohne Erfolg Lernenden gehören.

Der Versuch Geschlecht, Schulerfolg, soziale Schicht und biografische Bindungs- und Bildungserfahrung zusammenzudenken macht deutlich, dass Intersektionalität mit hohen Ansprüchen an die Bewältigung von Komplexität ein- 
hergeht. Wenn Leslie Mc Call (2005, S. 1772) schreibt: «In an nutshell, research practice mirrors the complexity of social life ...»), so lässt sich im Lichte der Perspektivitästheorie relativieren, dass jedes Bemühen, etwas von der Komplexität der kindlichen Lebenszusammenhänge einzufangen lobenswert ist und dennoch unvollkommen bleiben muss. Eben diese Unvollkommenheit bedingt die Bewegungen der einzelnen forschenden Person und die Bewegungen der Forschergemeinschaft mit ihren Paradigmenwechseln.

\section{Transnationalität und andere Transgressionen in disziplinären Feldern}

Mit der Vorsilbe "Trans...» wird angezeigt, dass beabsichtigt ist Übergänge zu untersuchen. Mit dieser Aufmerksamkeit für Bewegungen zwischen sozialen Räumen kommt eine zeitliche Dimension ins Spiel. Die Komplexität der vielfältig sich überschneidenden Heterogenitätsdimensionen wird um eine weitere Dimension, um einen Ausschnitt aus einem zeitlichen Verlauf, erweitert. In den folgenden Abschnitten kann gezeigt werden, dass Transgressionen auf vorher nachher, auf zeitliche Veränderungen, hinsichtlich verschiedenster Heterogenitätsdimensionen in unterschiedlichen perspektivischen Grössendimensionen ${ }^{4}$ bezogen werden, auf Nationen/Kulturen, auf Geschlechter sowie auf lebensgeschichtliche Altersstufen und Ereignisse.

Mit Transnationalität oder Transkulturalität wird ein globales Übergangsphänomen bezeichnet. Durch Wanderungen bis hin zu einem modernen Nomadentum entstehen transnationale oder transkulturelle Lebensentwürfe (Sting, 2006). Migration ist keineswegs neu. «Historisch gesehen, bildet Transkulturalität wohl eher die Regel als die Ausnahme. Wanderungsbewegungen, Kriege, Handelsbeziehungen aller Art haben schon in der archaischen Gesellschaft das eigene mit dem Fremden konfrontiert. Die Mythen und Religionen des östlichen Mittelmeerraums erzählen in verschiedenen Varianten davon» (Göhlich $e t$ al. 2006, S. 8). Wanderungsbewegungen und vielfältige Kulturen haben in der Geschichte des Schulwesens in allen Epochen eine Rolle gespielt (Krüger-Potratz, 2006). Den Herausgebern des Buches «Transkulturalität und Pädagogik» kommt das Verdienst zu, die Geschichte der permanenten Wanderungsbewegungen und ihren inzwischen Jahrhunderte währenden Niederschlag in philosophischen und pädagogischen Theorien bewusst zu machen. Der Begriff Transnationalität ist dem der Transkulturalität nahe, er akzentuiert etwas stärker die aktuellen Formen der Migration von Angehörigen verschiedener Nationen, er «bezeichnet Verflechtungen im ökonomischen, politischen, kulturellen und sozialen Bereich sowie die hierauf bezogenen Organisationsformen, die gesellschaftliche Formationen hervorbringen, die quer zu nationalstaatlichen und -gesellschaftlichen Grenzen verlaufen" (Homfeldt, Schröer \& Schweppe, 2006, S. 7). Einige Autoren legen Wert darauf, «Migrationspädagogik» von der «Interkultu- 
rellen Pädagogik» abzugrenzen, sie möchten u.a. betonen, dass «Migration» nicht nur Einwanderung umfasst, sondern auch das Auswandern, Weiterwandern oder Zurückkehren (vgl. z.B. Mecheril, 2004). Mit den Herausgebern und Autoren des oben erwähnten Bandes «Transkulturalität und Pädagogik» finde ich, dass der Begriff der "Kultur» und aus ihm gebildete Wortverbindungen eine für Kinder in Schulen hochbedeutsame Heterogenitätsdimension benennen (u.a. Wulf, 2006). "Transnationalität» und "Transmigration» heben andere Aspekte hervor und haben darum ihre Berechtigung, können aber den Kulturbegriff nicht ersetzen. Wenn es um Bildung geht, ist der Bezug zu Kultur unumgänglich. Die kognitiven, sozialen und gesellschaftlichen Funktionen der Schule haben mit zukunftsorientierten kulturellen Entwicklungen zu tun, der Begriff «interkulturelle Bildung» impliziert keineswegs per se einen statischen oder gar essentialistischen Kulturbegriff und muss die Aufmerksamkeit für transkulturelle Phänomene nicht ausschliessen.

Die Lebenszusammenhänge der Kinder mit Migrationshintergrund sind von den Migrationsbewegungen und den (sub-)kulturellen Zugehörigkeiten der Eltern geprägt. Zugleich entwickeln sie eigene Selbst- und Weltbilder, die in den Übergangssituationen, in denen sie leben, begründet sind (z.B. Diehm \& Kuhn, 2005; Mecheril, 2003). In der vielsprachigen Schweiz wurden früh didaktische Handlungsstrategien erprobt, die die migrationsgeprägten, besonderen Kompetenzen dieser Kinder auf allen Bildungsstufen nutzen (Schader, 2004).

In einem weiteren Feld, in der Geschlechterforschung, zeigen Begriffe wie «Transgender», "Transidentität», «Transsexualität» Grenzüberschreitungen zwischen der überkommenen weiblich-männlichen Dualität an. Als Transgender bezeichnen sich Menschen, für die die Zuweisung zu einem von zwei Geschlechtern, die sie nach ihrer Geburt erhalten haben, problematisch ist, das schliesst auch intersexuelle Menschen (Lang, 2006) ein. Sie bewegen sich auf vielfältige Weise zwischen Weiblichkeit und Männlichkeit und streben teilweise an, die Geschlechterdualität aufzulösen. Geschlechtergrenzen überschreitende Personen haben soziale und wissenschaftliche Initiativen gegründet, um ihren oft verdrängten, tabuisierten und stark abgelehnten Lebensweisen Gehör zu verschaffen und die Lebensbedingungen zu analysieren. Die Namen der Initiativen spiegeln die Vielfalt der Lebensweisen und sexuellen Orientierungen wider, so zum Beispiel die noch vergleichsweise einfache Abkürzung TrIQ, mit der sich das 2006 gegründete Beratungs- und Bildungsprojekt "TransInterQueer» bezeichnet oder beispielsweise LGBT (lesbian, gay, bisexual and transgender, vgl. Moorhead, 2005; LSVD Verbandstag 2002). Der Bundesarbeitskreis für Lesben, Schwule, Bisexuelle und Transgender in der Gewerkschaft ver.di nutzt zur abkürzenden Benennung des Vielfältigen das Wort «Regenbogen». Die OnlineZeitschrift «Liminalis - Zeitschrift für geschlechtliche Emanzipation» wird verantwortet vom wissenschaftlichen Beirat des Transgender Netzwerkes Berlin. Der Titel verweist mit dem auf Victor Turner (1964) zurückgehenden Begriff der «Liminalität»auf einen Schwebezustand bei einem Statuswechsel, auf die Mehr- 
deutigkeit, die ein solches Zwischenstadium auszeichnet. Mit dem Ansatz der "Queer-Theory» wurde eine die hier benannten vielfältigen Lebens- und Empfindungsweisen zusammenfassende und grundlegende Kritik der hierarchischen Dualitäten Mann - Frau sowie Heterosexualität - Homosexualität entwickelt (zusammenfassend Hartmann, 2004). Über die «Regenbogen»-Themen hinaus verweist der Ansatz «Gender in Motion» (Bankosegger \& Forster, 2007) auf ein von Bewegung bestimmtes Verständnis allgemeiner Geschlechterverhältnisse.

Die aus dem Konzept des Transgender und verwandten Ansätzen hervorgehende Pädagogik setzt auf den Schwebezustand. So propagiert Jutta Hartmann eine "Pädagogik vielfältiger Lebensweisen», in der es darum geht, "geschlechtliche und sexuelle Grenzen als beweglich anzuerkennen und optativ eine Vielzahl von Lebensweisen zu entfalten» (Hartmann, 2004, S. 266). Die Brüchigkeit von Geschlechtsidentität soll sichtbar gemacht werden, verdrängte Beispiele solcher Grenzüberschreitungen aus der Geschichte können aufklärend herangezogen werden. Nicht feste Identität soll intendiert werden, sondern Kinder und Jugendliche sollen angeregt werden zu einer «Ausarbeitung und Gestaltung der eigenen Identität, die bisherige Grenzen befragt, ausdehnt und zum Teil auch überschreitet» (Hartmann 2004, S. 264). Jutta Hartmann fundiert ihren Ansatz, indem sie Beispiele für Überschreitungen von Geschlechtergrenzen in der Schule aus der Kindheitsforschung, u.a. nach Anja Tervooren (2006) heranzieht.

Vor allem im Hinblick auf Übergänge im Lebenslauf wird der Begriff «Transition» (Welzer, 1993) verwendet. Er bezieht sich darum in der Regel stark auf die Heterogenitätsdimension «Alter» und die im Laufe des Älterwerdens wechselnden Lebensphasen sowie auf kritische Ereignisse im Lebenszusammenhang der Kinder, wie z.B. Ehescheidungen der Eltern. Die Gestaltung von Übergängen mit Übergangsriten wurde für die verschiedensten Kulturen und historischen Epochen untersucht (vgl. z.B. Van Gennep, 1986). Die Transitionstheorie versteht Transitionen als individuelle und soziale Prozesse, die sich innerhalb sich verändernder Kontexte abspielen. Intrapsychische, sozial-interaktive und durch äussere Lebensereignisse bedingte Übergänge fordern dazu heraus, sich an neue Situationen anzupassen, alte Werte in neue Orientierungen zu integrieren und zu neuen Entwürfen zu finden. «Mit dem Transitionskonzept wird es möglich, sowohl die individuelle Lebensgeschichte stärker zu berücksichtigen als auch aktuelle Entwicklungsschritte mit ihren vielfältigen Facetten in der Interaktion mit dem Kontext genauer zu verstehen» (Wörz, 2004, S. 28). "Transition wird», nach Thomas Wörz (2004, S. 36), «also auf Lebensereignisse bezogen, die eine Bewältigung von Veränderungen auf mehreren definierten Ebenen erfordern der individuellen, interaktionalen und kontextuellen - und in der Auseinandersetzung des einzelnen und seines sozialen Systems mit gesellschaftlichen Anforderungen Entwicklung stimulieren und als bedeutsame biografische Erfahrungen in der Identitätsentwicklung ihren Niederschlag finden».

Transitionstheoretische Anregungen sind in elementarpädagogischen Studien aufgegriffen worden. Übergänge von der Familie in die Krippe, von Familie oder 
Krippe in den Kindergarten, vom Kindergarten in die Schule werden als Transitionen analysiert (Griebel \& Niesel, 2004). Die Befunde von Pamela Oberhuemer (2004) machen deutlich, dass sich international zwei Zielrichtungen der den Übergang in die Schule vorbereitenden Pädagogik unterscheiden lassen. Die eine Richtung betont spielendes, individuelles und soziales Lernen (u.a. Schweden, Dänemark, Deutschland, deutschsprachige Schweiz), die andere Richtung betont schulbezogenes fachliches Lernen (u.a. Frankreich, Grossbritannien, Niederlande und die französischsprachige Schweiz). Im Zentrum einer wichtigen Innovation im schweizerischen Bildungswesen, der Basisstufe, steht die Transition vom Elementar- zum Primarbereich (Wannack, 2006). Die Mischung der Altersgruppen ist kennzeichnend für die Innovationen am Übergang vom Kindergarten zur Schule (Boes, 2002).

Die mit Transnationalität (oder -kulturalität), Transgender und Transition arbeitenden Ansätze haben gemeinsam, dass sie sich auf Menschen in Übergangsund Überschneidungssituationen beziehen, in ihren schulpädagogischen Anwendungen machen sie auf Kinder in Schwebezuständen und Zwischenräumen aufmerksam. Sie berücksichtigen die Heterogenitätsdimensionen Nation bzw. Kultur, Geschlecht und Lebensalter und stellen damit Kategorien für Analysen und Handlungskonzepte bereit. Zu den Übergangswegen jedes Kindes, jeder Kindergruppe, in Schulen können aus der Perspektive dieser Heterogenitätsdimensionen Einsichten gewonnen werden. Überblendet man die Dimensionen im Sinne von Intersektionalität, entsteht ein sehr komplexes Verständnis für Kollektivität und Einzigartigkeit kindlicher Lebens- und Bildungswege.

\section{Pädagogik der Vielfalt: Weiterentwicklung, Forschung und Ausbildung}

In der Pädagogik der Vielfalt sind Transgressionsphänomene nicht neu, Veränderlichkeit der lebensgeschichtlichen und der biografischen Entwicklungen gehört zu dem für sie grundlegenden Begriff von Differenz (z.B. Prengel, 2006, S. 182) und Heterogenität (Heinzel \& Prengel 2002, S. 12). Auch ist für sie kennzeichnend, dass sie sich nicht nur auf eine, sondern - wie der Name sagt grundsätzlich auf vielfältige Heterogenitätsdimensionen bezieht. Die Unvollständigkeit und Veränderlichkeit des Erkenntnisprozesses selbst und damit ein Verzicht auf rechthaberische Distanzierung von früheren Erkenntnissen gehört unverzichtbar dazu (Prengel, 2006, S. 51). So wurde die während der Bildungsreform vorangehende Betonung von Chancengleichheit als unabdingbare Basis der Pädagogik der Vielfalt anerkannt und in das Konzept der egalitären Differenz integriert. Auch die Schwierigkeiten, der Vielfalt pädagogisch gerecht werden zu können, die «Illusionen der Vielfalt», wurden zur Diskussion gestellt (Prengel, 1999). Hier wurde herausgearbeitet, dass jeder pädagogische Ansatz historischen und kulturellen Begrenzungen verhaftet ist. Vielfalt kann darum in 
der Pädagogik niemals uneingeschränkt erreicht werden. Wichtig ist, dennoch für Vielfalt offen zu sein und zugleich Begrenzungen, wie zum Beispiel Leistungsnormen, die in pädagogischen Ansätzen enthalten sind, transparent zu machen. Die in den vorangehenden Abschnitten behandelten Diskurse setzen zugleich auch Akzente, die die Pädagogik der Vielfalt weiterbringen können. So wurde die Einsicht, dass mit verschiedenen Heterogenitätskategorien «existentielle Dimensionen jeder Lebensgeschichte thematisiert» (Prengel, 2006, S. 14) werden, von Anfang an thematisiert, sie lässt sich aber auf dem Hintergrund der inzwischen weiter entwickelten Intersektionalitätstheorien konsequenter für Analysen nutzen. Auch können die in diesem Beitrag vorgestellten Transgressionsansätze dazu anregen, die gesellschaftlich bedeutender gewordenen Bewegungen der Menschen über Grenzen aller Art hinweg stärker zu berücksichtigen und Schwebezuständen noch mehr Aufmerksamkeit zu schenken als vor 20 Jahren, zur Zeit der Anfänge der Pädagogik der Vielfalt. Pädagogik der Vielfalt hat «Alter» mit seinen lebensgeschichtlichen Transitionen als Heterogenitätsdimension bisher meines Wissens wenig beachtet. Eine Ausnahme bildet aber das Thema "Jahrgangsmischung» (Prengel, 2007b). Das Transitionskonzept ist geeignet die Pädagogik der Vielfalt in ihrer Offenheit für Veränderungen in der Zeit zu bestärken, auch kann es mit seiner Aufmerksamkeit für Ko-Konstruktionen im systemischen Kontext die Pädagogik der Vielfalt bereichern.

Für erziehungswissenschaftliche Forschungen stellen Transgressionen Herausforderungen dar: Es ist schwierig in theoretischen und empirischen Vorhaben die Dynamik beider Seiten, der Forschenden und der Kinder in einer bestimmten Zeitspanne einzufangen und dabei vielfältig sich überschneidende Heterogenitätsdimensionen zu berücksichtigen. Nach Massgabe der jeweiligen Forschungsfrage ist es notwendig und berechtigt die komplexe Heterogenität zu reduzieren und nur ausgewählte Differenzkategorien zu untersuchen, aber kategoriale Zuschreibungen durch Forschung sind riskant, denn sie können sich wie Vorurteile auswirken, die Kinder unpassenden Vorstellungen subsumieren.

Es gibt Wege in der Forschung, solche Gefahren des etikettierenden Zuordnens und der Pauschalisierung zu vermeiden. Kategoriale Begrenzungen und ihre Folgen sollten in Forschungsvorhaben beschrieben, begründet und mit ihren Folgen reflektiert werden. Nach Möglichkeit sollten mehrere Heterogenitätsdimensionen berücksichtigt werden. Wenn man in der Kindheits- und Schulforschung zum Beispiel Geschlecht, Kultur und ökonomischen Status analysiert, hat man die Chance Ursachen von Schulerfolg und Schulversagen gründlicher zu erfassen als wenn man Daten nur binär-zweigeschlechtlich unterteilt und die Kinder so dual pauschalisierend in schulversagende Jungen und schulerfolgreiche Mädchen einteilt (vgl. Andresen, 2008). Im Sinne der Aufmerksamkeit für Schwebezustände ist es weiterführend nach Kindern im Übergang zwischen den Kulturen, den Geschlechtern und Leistungsgruppen zu fragen.

Eine weitere Möglichkeit Vorurteile durch Forschung zu vermeiden ist, anhand eines "präkategorialen» Forschungszugangs (Bührmann, 2008), der weit- 
gehend darauf verzichtet selbst kategorial zuzuordnen, die Kinder selbst auf ihre eigenen kategorialen Konstruktionen hin zu befragen oder zu beobachten (vgl. Faulstich-Wieland, Weber \& Willems, 2004). Nur so ist es angesichts unübersichtlicher dynamischer transkultureller Lebenswelten möglich etwas darüber zu erfahren, wie Kinder Transgressiosprozesse individuell erleben und wie sie sich selbst in ihnen verorten, sei es in der Nähe einer Herkunftskultur, sei es in der Nähe der Kultur eines Einwanderungslandes, sei es in der Nähe neuer kultureller Formationen in multikulturellen Gesellschaften. Durch Kinderforschung ist es möglich auch über jene Wünsche und Lebensentwürfe als geschlechtliche Wesen Informationen zu erhalten, die nicht in zweigeschlechtlichen Kontrasten stecken bleiben, sondern der Tatsache gerecht werden, dass es zum Beispiel Mädchen und Jungen gibt, die im Sinne von Transgender gleichgeschlechtliche Neigungen (Schmauch, 2008) oder dem Klischee zuwiderlaufende Interessen (MacNaughton, 2004) entwickeln.

Auch für die Ausbildung von Pädagoginnen und Pädagogen im Elementar-, Primar- und Sekundarbereich stellen Transgressionen im Lebenszusammenhang der Kinder und Jugendlichen Herausforderungen dar. Sie bilden ein wichtiges Thema für den Studienbereich der Querschnitts- bzw. Schlüsselqualifikationen. Hochschulen haben an mehreren Orten begonnen «Heterogenität» zu einem Leitbegriff ihrer Profile zu machen (z.B. Bräu \& Schwerdt, 2005). Für pädagogische Ausbildungsgänge an Fachhochschulen und Universitäten werden Heterogenitäts- und Diversity-Module 5 entworfen. Ziel der Ausbildung in neuen Bachelor-Studiengängen für den Erzieherberuf an Fachhochschulen und Universitäten hinsichtlich des «Diversity» genannten Moduls ist es beispielsweise, dass Studierende ein grundlegendes Verständnis der Pädagogik der Vielfalt und darauf bezogene Kompetenzen «für die Arbeit mit Kindern und Eltern, für die Selbstreflexion, für die Teamarbeit, für die Qualitätsentwicklung der Einrichtung sowie für die Vernetzung im Sozialraum entwickeln. Die Aneignung von Wissen über Formen des gesellschaftlichen Umgangs mit Vielfalt, über die Zusammenhänge zwischen sozialer Ungleichheit und Bildungschancen sowie über Inklusions- und Exklusionsprozesse in Bezug auf das Erziehungs- und Bildungssystem bildet ein weiteres Ziel dieses Moduls» (Prengel \& Steinhilber 2007, S. 1f). Der Bachelor-Studiengang soll auf drei Ebenen qualifizieren: Er soll neben der Vermittlung von grundlegendem Wissen und von Handlungsmöglichkeiten dazu beitragen, dass die Studierenden den persönlichen und professionellen Umgang mit Verschiedenheit reflektieren können. Wenn in diesem Ausbildungsprogramm die Fähigkeit der Reflexion und Selbstreflexion betont wird, wird den Studierenden die Aufmerksamkeit für Schwebezustände nahe gebracht, sie haben eine Chance etwas darüber zu lernen, in welchen Unsicherheiten sich Kinder in transnationalen Gesellschaften bewegen und feinfühlig darauf einzugehen.

Pädagogische Ausbildungsgänge sollten allesamt dazu befähigen, dass die angehenden PädagogInnen lernen, Lebenszusammenhängen und Bezugspersonen 
(Griebel \& Niesel, 2004) ihrer Adressaten einzubeziehen und eine verlässliche «Schule als Lebenswelt» (Grunder, 2006) in Ansätzen zu gestalten, weil sie Kindern angesichts der Unsicherheit von Übergangssituationen Halt geben können. Die Evaluation dieser Ausbildungsansätze ist ein Forschungsdesiderat.

Die Aufmerksamkeit für Transgressionen in der Lebenswelt der Schülerinnen und Schüler steigert die Notwendigkeit, komplexe Zusammenhänge in ihrer zeitlichen Veränderlichkeit zu denken. Nicht nur die Fähigkeit zum Perspektivenwechsel, sondern darüber hinaus auch die Fähigkeit zum dezentrierenden Gleiten der Perspektiven bilden hohe Anforderungen an Studierende und Dozierende. Angesichts der Unübersichtlichkeit der gleichzeitig wirksamen Heterogenitäts- und Transgressionsphänomene bilden Menschenrechte, Menschenrechtsbildung (Overwien \& Prengel, 2007) und ihre Konkretisierung in den vielen Erfindungen demokratischer Pädagogik (Geiling \& Heinzel, 2004) eine sichere Orientierung und Massstäbe zur Beurteilung von Ansätzen der DiversityEducation aller Art. In Ausbildungsgängen kann eine Einführung in die menschenrechtlich begründeten Prinzipien der Gleichheit und Freiheit für Verschiedenheit eine Grundlage schaffen für die Auseinandersetzung mit der Fülle konkreter praxisbezogener Konzepte, die sich auf einzelne Heterogenitätsdimensionen, Zielgruppen und Arbeitsfelder beziehen.

\section{Anmerkungen}

1 In diesem Beitrag ist, wenn es um die Adressaten der Schule geht, vor allem von Kindern die Rede. Der Text impliziert partiell auch Jugendliche.

2 Transgression wird hier als Oberbegriff für Übergänge hinsichtlich verschiedener Heterogenitätsdimensionen verwendet.

3 Erstaunlich, auch schade, ist, dass es bildungswissenschaftliche Sammelbände zum Thema «Heterogenität» gibt, deren Herausgeber vollständig auf eine theoretische Klärung ihres Themas verzichten, so Bos, Lankes \& Plassmeier (2004).

4 Die Analyse von Transgressionen im schulpädagogisch wichtigen Feld der Leistungsvielfalt und der Inklusiven Pädagogik als Arbeit mit behinderten und nichtbehinderten Kindern sowie eine ausführliche Berücksichtigung der Dimension des sozioökonomischen Status müssen späteren Studien vorbehalten bleiben. Vgl. dazu z.B. Hinz, 1998.

5 Die in Nordamerika auf der Basis der Bügerrechtsbewegungen entstandene und gegenwärtig international rezipierte «Diversity-Education» weist grundlegende Gemeinsamkeiten mit der in Europa entstandenen «Pädagogik der Vielfalt» auf, dazu gehören das Wertschätzen von Pluralität und die Orientierung an Gleichheit und Freiheit im Sinne der Menschenrechte, vgl. zusammenfassend.

\section{Literaturverzeichnis}

Andresen, S. (2008). Kinder und soziale Ungleichheit. Ergebnisse der Kindheitsforschung zu dem Zusammenhang von Klasse und Geschlecht. In A. Prengel \& B. Rendtorff (Hrsg.), Kinder und ihr Geschlecht. Jahrbuch Frauen- und Geschlechterforschung in der Erziehungswissenschaft (Bd. 4). Opladen und Farmington Hills: Barbara Budrich.

Bankosegger, K. \& Forster, E. (Hrsg.). (2007). Gender in Motion. Genderdimensionen der Zukunftsgesellschaft. Wiesbaden: VS. 
Becker, U. (2007). Schuldistanz - eine Herausforderung für die Pädagogik. Habilitationsvortrag, gehalten am 5.7.2007 an der Universität Potsdam.

Bielefeldt, H. (1998). Philosophie der Menschenrechte. Grundlage eines weltweiten Freiheitsethos. Darmstadt:WBG.

Boes, A. (2002). Jahrgangsübergreifende Lerngruppen. Paradigma für eine Schule der Zukunft. Berlin. [http://www.gew-berlin.de/documents_public/030224_AdBoesReferat.pdf]. (Stand 8.7.2007).

Bos, W., Lankes, E.-M. \& Plassmeier, N. (2004): Heterogenität. Eine Herausforderung an die empirische Bildungsforschung. Münster: Waxmann.

Bräu, K. \& Schwerdt, U. (Hrsg.). (2005). Heterogenität als Chance. Vom produktiven Umgang mit Gleichheit und Differenz in der Schule. Münster u.a.: LIT.

Bührmann, A. (2008). Perspektiven der Intersektionalitätsforschung. Vortrag, Universität Potsdam, 12.1.2008.

Diehm, I. \& Kuhn, M. (2005). Ethnische Unterscheidungen in der frühen Kindheit. In Hamburger, F. (Hrsg.), Migration und Bildung (S.221-231). Wiesbaden: VS.

Fuchs, M. (1999). Kampf um Differenz. Repräsentation, Subjektivität und soziale Bewegungen. Das Beispiel Indien. Frankfurt a.M.: VS.

Faulstich-Wieland, H., Weber, M. \& Willems, K. (2004). Doing Gender im heutigen Schulalltag. Empirische Studien zur sozialen Konstruktion von Geschlecht in schulischen Interaktionen. Weinheim und München: Juventa.

Geiling, U. \& Heinzel, F. (Hrsg.). (2004). Demokratische Perspektiven in der Pädagogik. Weinheim: VS.

Glaser, E., Klika, D. \& Prengel, A. (2004). Handbuch Gender in der Erziehungswissenschaft. Bad Heilbrunn: Klinkhardt.

Göhlich, M., Leonhard, H. W., Liebau, E. et al. (Hrsg.). (2006). Transkulturalität und Pädagogik. Interdisziplinäre Annäherung an ein kulturwissenschaftliches Konzept und seine pädagogische Relevanz. Weinheim und München: Juventa.

Graumann, C. F. (1960). Grundlagen einer Phänomenologie und Psychologie der Perspektivität. Berlin: De Gruyter.

Graumann, C. F. (2002). Toleranz und Perspektivität. In F. Heinzel \& A. Prengel, (Hrsg.), Heterogenität, Integration und Differenzierung in der Primarstufe (S. 22-30). Opladen: Leske \& Budrich.

Griebel, W. \& Niesel, R., mit Beiträgen von T. Wörz und U. Meiser (2004). Transitionen. Fähigkeit von Kindern in Tageseinrichtungen fördern, Veränderungen erfolgreich zu bewältigen. Weinheim und Basel: Beltz.

Grunder, H.-U. (2006). Schule und Lebenswelt, Schule oder Lebenswelt, Schule als Lebenswelt. Anmerkungen zu einem gespannten Verhältnis. Päd Forum: Unterrichten und Erziehen 34./25. (3), 182-185.

Hartmann, J. (2004). Dekonstruktive Perspektiven auf das - Referenzsystem von Geschlecht und Sexualität - Herausforderungen der Queer-Theory. In E. Glaser, D. Klika \& A. Prengel (Hrsg.), Handbuch Gender in der Erziehungswissenschaft (S. 255-271). Bad Heilbrunn: Klinkhardt.

Heinzel, F. \& Prengel, A. (2002). Heterogenität, Integration und Differenzierung in der Primarstufe. Opladen: Leske \& Budrich.

Hinz, A. (1998). Pädagogik der Vielfalt auch für Schulen in Armutsgebieten? Überlegungen zu einer theoretischen Weiterentwicklung In A. Hildeschmidt \& I. Schnelle (Hrsg.), Integrationspädagogik. Auf dem Weg zu einer Schule für alle (S. 127-144). Weinheim/München: Juventa.

Homfeldt, H. G., Schröer W. \& Schweppe, C. (2006). Transnationalität, soziale Unterstützung, agency (S. 7). Nordhausen: Bautz.

Klinger, C., Knapp, G.-A. \& Sauer, B. (Hrsg.). (2007): Achsen der Ungleichheit. Zum Verhältnis von Klasse, Geschlecht und Ethnizität. Wiesbaden: Campus. 
Knapp, G.-A. (2005). Race, Class, Gender. Reclaiming Baggage in Fast Travelling Theories. European Journal of Women's Studies 12 (3), 249-265.

Koller, H.-C. (2002): Bildung und kulturelle Differenz. Zur Erforschung biografischer Bildungsprozesse von MigrantInnen. In M. Kraul \& W. Marotzki (Hrsg.), Biographische Arbeit. Perspektiven erziehungswissenschaftlicher Biographieforschung (S. 92-116). Opladen: Leske \& Budrich.

Krüger-Potratz, M. (2006): Präsent - aber «vergessen» - Zur Geschichte des Umgangs mit Heterogenität im Bildungswesen. In M. Göhlich et al. (Hrsg.), Transkulturalität und Pädagogik. Interdisziplinäre Annäherung an ein kulturwissenschaftliches Konzept und seine pädagogische Relevanz (S. 212-137). Weinheim und Müchen: Juventa.

Lang, C. (2006). Intersexualität. Menschen zwischen den Geschlechtern. Frankfurt/NewYork: Campus.

Liminalis - Zeitschrift für geschlechtliche Emanzipation 2007 (1) [http://www.liminalis.de/]. (Stand 29.7.2007).

LSVD Verbandstag (2002). Diskriminierung von Transgender beenden. Grundsatzpapier. Köln: [http://typo3.lsvd.de/286.0.html]. (Stand 29.7.2007).

Lutz, H. \& Wenning, N. (Hrsg.). (2001). Unterschiedlich verschieden. Differenz in der Erziehungswissenschaft. Wiesbaden: Leske + Budrich.

MacNaughton, G. (2004). Gender - neu gedacht in der Pädagogik der frühen Kindheit. In E. W. Fthenakis \& P. Oberhuemer (Hrsg.), Frühpädagogik international. Bildungsqualität im Blickpunkt (S. 345-355). Wiesbaden: Leske + Budrich.

Mc Call, L. (2005). The Complexity of Intersectionality. Signs, Journal of Women in Culture and Society 30 (3), $1771-1802$.

Mecheril, P. (2004). Einführung in die Migrationspädagogik. Weinheim und Basel: Beltz.

Mecheril, P. (2003). Prekäre Vehältnisse. Über natio-ethno-kulturelle (Mehrfach) Zugehörigkeit. Münster: Waxmann.

Moorhead, C. (2005). "Advising lesbian, gay, bisexual, and transgender students in higher education». NACADA Clearinghouse of Academic Advising Resources. Online: [http://www.nacada.ksu.edu/Clearinghouse/AdvisingIssues/LGBTA.htm]. (13.6.2007).

Oberhuemer, P. (2004). Bildungskonzepte für die frühen Jahre in internationaler Perspektive. In W.E. Fthenakis \& P. Oberhuemer (Hrsg.), Frühpädagogik international. Bildungsqualität im Blickpunkt (S. 359-383). Wiesbaden: Leske + Budrich.

Overwien, B. \& Prengel, A. (2007). Recht auf Bildung. Zivilgesellschaftliche Stimmen zum Besuch von Venor Muñoz in Deutschland. Opladen und Farmington Hills: Barbara Budrich Verlag.

Prengel, A. (unter Mitarbeit von Heinzel, F., Geiling, U. \& Hemme-Kreutter, M.). (1999). Vielfalt durch gute Ordnung im Anfangsunterricht. Opladen: Leske + Budrich.

Prengel, A. (2006, zuerst 1993). Pädagogik der Vielfalt. Verschiedenheit und Gleichberechtigung in Interkultureller, Feministischer und Integrativer Pädagogik. Wiesbaden: VS.

Prengel, A. (2007a). Diversity-Education. Grundlagen und Probleme der Pädagogik der Vielfalt. In G. Krell, B. Riedmüller, B. Sieben \& D. Vinz (Hrsg.), Diversity Studies (S. 49-67). Frankfurt a.M.: Campus.

Pregel, A. (2007b). Heterogenität als Chance. In H. de Boer, K.-H. Burk \& F. Heinzel (Hrsg.), Lehren und Lernen in Jahrgangsgemischten Klassen (S. 66-75). Frankfurt a.M.: Grundschulverband - Arbeitskreis Grundschule.

Prengel, A. \& Steinhilber, B. (unter Mitarbeit von D. Benckendorff). (2007). Modul Diversity. PIK - Profis für Kitas, Programm zur Professionalisierung von Frühpädagogen in Deutschland (S. 1f). Bosch-Siftung und Alice-Salomon-Fachhochschule. Unv. Ms. Berlin.

Schader, B. (2004). Sprachenvielfalt als Chance. Hintergründe und 101 praktische Vorschläge für den Unterricht in mehrsprachigen Klassen. Troisdorf und Zürich: Orell Füssli.

Schmauch, U. (2008). Gleichgeschlechtliche Entwicklungen von Mädchen und Jungen. In A. Prengel \& B. Rendtorff (Hrsg.), Kinder und ihr Geschlecht. Jahrbuch Frauen- und Ges- 
chlechterforschung in der Erziehungswissenschaft (Bd. 4). Opladen und Farmington Hills: Barbara Budrich.

Sting, S. (2006). Migration, transkulturelle Räume und kollektive Identitäten. In M.Göhlich, , H.-W. Leonhard, E. Liebau, J. Zirfas (Hrsg.), Transkulturalität und Pädagogik. Interdisziplinäre Annäherung an ein kulturwissenschaftliches Konzept und seine pädagogische Relevanz (S. 45-55). Weinheim und München: Juventa.

Tenorth, H.-E. (2004). Erziehungswissenschaft. In D.Benner \& J. Oelkers (Hrsg.), Historisches Wörterbuch der Pädagogik (S. 341-382). Weinheim und Basel: Beltz.

Tervooren, A. (2006). Im Spielraum zwischen Geschlecht und Begehren. Ethnografie der ausgehenden Kindheit. Weinheim und München: Juventa.

TrIQ TransInterQueer, Berlin 2006. Online: [http://www.transinterqueer.org/]. (Stand 29.7.2007).

Turner, V. (1964). Betwixt and Between: The Liminal Period in Rites de Passage. In M. E. Spiro (Ed.), Symposium on New Approaches to the Study of Religion (p. 4-20). Seattle: American Ethnological Society.

Van Gennep, A. (1986). Übergangsriten. Frankfurt: Campus. (Original: Les rites de passage, Paris 1909).

Wannack, E. (2006). Eine Bildungsstufe für 4-8-jährige Kinder. Pädagogisch-didaktische Herausforderung oder Überforderung? Vortrag Solothurn. http://www.evelyne-wannack.ch/doc/referat_solothurn_06.pdf (Stand 25.2.2008).

Welzer, H. (1993). Transitionen. Zur Sozialpsychologie biografischere Wandlungsprozesse. Tübingen: edition diskord.

Wigger, L. (2003). Wie ist Bildung möglich? Vierteljahrsschrift für Wissenschaftliche Pädagogik 80 (1), 478-493.

Wörz, T. (2004). Die Entwicklung der Transitionsforschung. In W. Griebel \& R. Niesel (mit Beiträgen von T. Wörz und U. Meiser) (Hrsg), Transitionen. Fähigkeit von Kindern in Tageseinrichtungen fördern, Veränderungen erfolgreich zu bewältigen (S. 22-41). Weinheim und Basel: Beltz.

Wulf, C. (2004). Anthropologie, pädagogische. In D. Benner \& J. Oelkers (Hrsg.), Historisches Wörterbuch der Pädagogik (S. 33-57). Weinheim und Basel: Beltz.

Wulf, C. (2006). Heterologisches Denken und Handeln als Aufgabe transkultureller Bildung. In M. Göhlich, H.-W. Leonhard, E. Liebau, J. Zirfas (Hrsg.), Transkulturalität und Pädagogik. Interdisziplinäre Annäherung an ein kulturwissenschaftliches Konzept und seine pädagogische Relevanz (S. 155-168). Weinheim und München: Juventa.

Schlagworte: Diversity Education, Pädagogik der Vielfalt, Transgression, Transgender, Perspektivität, Lehrer/innenbildung, Elementarstufe 


\section{État incertain: Écoles et contextes de vie transgressifs}

\section{Résumé}

Dans les débats en sciences de l'éducation à propos de l'interculturel et de sa signification pour l'école et la formation des enseignants, l'attention pour des changements dynamiques et continus croît. La migration est moins pensée comme un pas précis du déplacement d'un pays d'origine vers une nouvelle patrie que comme un état incertain: les humains immigrent et poursuivent leur immigration ce qui amène les enfants et les jeunes à définir leur vie comme «un état entre deux».

La contribution met en évidence diverses approches théoriques, empiriques et pédagogiques relatives aux discussions en sciences de l'éducation à propos de la transnationalité. Des références à d'autres champs disciplinaires (p. ex. les «genres» et les «transitions» dans le système éducatif) sont établis. Finalement, seront présentées des conclusions en faveur du développement d'une «pédagogie de la diversité» et de sa concrétisation dans la recherche et la formation des enseignants du degré élémentaire.

Mots clés: interculturel, migration, pédagogie, transnationalité, formation des enseignants, degré élémentaire

\section{In bilico tra scuola e vita in transizione. Approcci nella ricerca e nell'insegnamento.}

\section{Riassunto}

Nel dibattito sull'interculturalità e il suo significato per la scuola e la formazione degli insegnanti cresce l'attenzione per processi di trasformazione dinamica. La migrazione vine concepita più come uno stato dinamico che non un passaggio delimitabile dal paese d'origine al paese ospitante: le persone vanno e vengono, $\mathrm{i}$ bacini e i giovani definiscono il loro modo di essere come qualcosa in transizione. Il contributo presenta aspetti teorici, empirici e pratici della discussione nelle scienze dell'educazione che toccano la transnazionalità. Vengono poi tracciate relazioni con altri campi disciplinari (ad es. "transgender", "transizioni" nel sistema formativo) per concludere con delle conclusioni per lo sviluppo di una "pedagogia della diversità" e la sua concretizzazione nella ricerca e nella formazione degli insegnanti del settore elementare.

Parole chiave: pedagogia della diversità, formazione degli insegnanti, educazione alla diversità, transizione 


\section{In a ufloating state": Schools and transgressive communities Summary}

Educational discourses with regards to intercultural life and its meaning for schooling and teacher training focus increasingly on processes of continued dynamic change. Migration is not as much to be understood as a distinctive move from the native country to a new home country than as an ongoing "floating state": People move in and move on; children perceive their lives as "in between". This contribution introduces theoretical, empirical, and practical approaches to the academic discourse of transnationalism. Relationships with other disciplinary fields (such as "transgender", and "transitions" in educational systems) are established. Conclusions with regards to further develop a "pedagogy of multiplicity", and related concrete concepts for research and teacher training (beginning as early as elementary level), are drawn.

Key words: schools, transgressive communities, transnationalism, pedagogy of multiplicity 